\title{
Consequences of the Continuity of the Monic Integer Transfinite Diameter
}

\author{
Jan Hilmar
}

February 9, 2020

\begin{abstract}
We consider the problem of determining the monic integer transfinite diameter

$$
t_{M}(I)=\lim _{n \rightarrow \infty} \inf _{p_{n} \in \mathcal{M}_{n}}\left\|p_{n}\right\|_{I}^{1 / n}
$$

for real intervals of length less than 4 . We show the $t_{M}([0, x])$, as a function in $x>0$, is continuous, therefore disproving two conjectures due to Hare and Smyth. Consequently, for $n>2 \in \mathbb{N}$, we define the quantity

$$
b_{\max }(n)=\sup _{b>\frac{1}{n}}\left\{b \mid t_{M}([0, b])=\frac{1}{n}\right\}
$$

and give lower and upper bounds of $b_{\max }(n)$. Finally, we use the algorithm from 4 to computationally improve this lower bound for $b_{\max }(n)$ for $3 \leq n \leq 8$.
\end{abstract}

\section{Introduction}

Let $I \subset \mathbb{R}$ be a closed interval of length less than 4 and $\mathcal{M}_{n}[x]$ be the set of monic polynomials of degree $n$ with integer coefficients. We define the monic integer transfinite diameter $t_{M}(I)$ of $I$ to be the quantity

$$
t_{M}(I)=\lim _{n \rightarrow \infty} \inf _{p_{n} \in \mathcal{M}_{n}}\left\|p_{n}\right\|_{I}^{1 / n}
$$

Here, $\left\|p_{n}\right\|_{I}=\sup _{x \in I}\left|p_{n}(x)\right|$ is the supremum norm of the polynomial $p_{n}(x)$. The problem of determining the monic integer transfinite diameter was first tackled by Borwein, Pinner and Pritsker in 3. Their techniques were further developed by Hare and Smyth in [4. The problem is intimately connected to the problem of determining $t_{\mathbb{Z}}(I)$, the integer transfinite diameter, where the condition that the polynomials be monic is removed. Interestingly, removing this condition makes the problem much harder, as no exact values of $t_{\mathbb{Z}}(I)$ are known, but $t_{M}(I)$ can be computed explicitly in some cases.

The following lemma is an essential tool in doing so:

Lemma $1([3])$. Let $q(x)=a_{0}+\cdots+a_{d} x^{d} \in \mathbb{Z}_{n}[x]$ be an irreducible polynomial with $a_{d}>1$ and all roots in the closed interval $I \subset \mathbb{R}$ of finite length. Further, assume that $p_{n}(x) \in \mathcal{M}_{n}[x]$. Then

$$
a_{d}^{-\frac{1}{d}} \leq\left\|p_{n}\right\|_{I}^{\frac{1}{n}}
$$


The proof of this essentially classical result can be found in [3] or [4] and will be omitted here.

As a consequence, $a_{d}^{-1 / d} \leq t_{M}(I)$, so that polynomials $q(x)$ as in the lemma are used to determine lower bounds on $t_{M}(I)$. As a consequence, they are called obstruction polynomials for $t_{M}(I)$ with obstruction $a_{d}^{-1 / d}$. Since all obstructions give a lower bound, it is of interest to find the supremum

$$
m(I)=\sup \left\{a_{d}^{-1 / d} \mid q(x)=a_{d} x^{d}+\cdots+a_{0}, a_{d}>1\right\} .
$$

Here the supremum is taken over all polynomials with integer coefficients and all roots in the interval $I$.

If the supremum is attained, $m(I)$ is called the maximal obstruction for $I$.

Suppose now we have an interval $I$ with maximal obstruction $m(I)$ and find a $p_{n}(x) \in \mathcal{M}_{n}$ with $\left\|p_{n}\right\|_{I}=m(I)^{n}$. In this case, $m(I) \geq t_{M}(I) \geq m(I)$, so that we have determined an exact value for $t_{M}(I)$. Such $p_{n}(x)$, if it exists, is said to attain the maximal obstruction. Some examples of this situation are as follows:

1. If $I=[0,1], \frac{1}{2}$ is the maximal obstruction by $q(x)=2 x-1$. At the same time, $\|x(1-x)\|_{I}=\frac{1}{4}$, so that $t_{M}(I)=\frac{1}{2}$.

2. For an integer $n>1$, consider $I_{n}=\left[0, \frac{1}{n}\right]$. $\frac{1}{n}$ is the maximal obstruction by $q(x)=n x-1$. At the same time, $\|x\|_{I_{n}}=\frac{1}{n}$, so that $t_{M}\left(I_{n}\right)=\frac{1}{n}$.

These are just some examples to illustrate the technique. A more complete list of known values of $t_{M}(I)$ can be found in 3 , and [4].

It was shown in [4] that the maximal obstruction is not always attained by some $p_{n}(x)$ and explicit conditions for when it cannot be attained were given. The authors conjecture, however, that $t_{M}(I)=m(I)$ for all $I$. That this is not the case is a consequence of the continuity of a particular function, proved in section 2 ,

\section{Continuity of $t_{M}(x), x \geq 0$}

In [4, the authors consider intervals of the form $I=[0, b], \frac{1}{n}<b<\frac{1}{n-1}, n>$ $1 \in \mathbb{N}$. From $q(x)=n x-1$, we know that $t_{M}([0, b]) \geq \frac{1}{n}$ and equality holds in a neighbourhood to the right of $\frac{1}{n}$ (see Theorem 9.1 in [4]). Much more interesting is the behaviour of the function

$$
t_{M}(x)=t_{M}([0, x]), x \geq 0
$$

to the left of $\frac{1}{n}, n>1$. Hare and Smyth suspected the function to have a discontinuity at $x=\frac{1}{n}$, which would agree with their conjecture that $m(I)=$ $t_{M}(I)$.

To answer this question, one has to look back at the classical paper [2] of Borwein and Erdélyi in the theory of the (non-monic) transfinite diameter. In this paper, the authors define the function $t_{\mathbb{Z}}(x)$ in the equivalent way and state that this function is continuous, though without the details of the proof. We will now provide the details for $t_{M}(x)$.

Let $T_{n}(x)$ be the $n^{\text {th }}$ Chebyshev polynomial on $[-1,1]$, defined by

$$
T_{n}(x)=\cos (n \arccos x)
$$


This can be rewritten as

$$
T_{n}(x)=\frac{1}{2}\left[\left(x+\sqrt{x^{2}-1}\right)^{n}+\left(x-\sqrt{x^{2}-1}\right)^{n}\right] .
$$

From this it immediately follows that

$$
T_{n}(x) \leq\left(x+\sqrt{x^{2}-1}\right)^{n} \text { for } x \geq 1 .
$$

We will also need Chebyshev's inequality from [1]:

Lemma 2. Let $p \in \mathbb{R}[x]$. Then, for $y \in \mathbb{R} \backslash[-1,1]$,

$$
|p(y)| \leq\left|T_{n}(y)\right|\|p\|_{[-1,1]}
$$

We can then prove:

Lemma 3. Let $b>b_{0}>0, p_{n} \in \mathbb{R}_{n}[x]$. Then, for every $\delta>0$, there exists $k_{b, \delta}$, not depending on $n$, such that

$$
\left\|p_{n}\right\|_{[0, b+\delta]} \leq\left(1+k_{b, \delta}\right)^{n}\left\|p_{n}\right\|_{[0, b]}
$$

with $\lim _{\delta \rightarrow 0} k_{b, \delta}=0$ for fixed $b$.

Proof. Given $p \in \mathbb{R}_{n}[x]$, let $y \in[0, b]$. Then $x=\frac{2}{b} y-1 \in[-1,1]$. Let

$$
q_{n}(x)=p_{n}\left(\frac{b}{2}(x+1)\right) .
$$

Then, for $x \notin[-1,1], y \notin[0, b]$,

$$
\begin{aligned}
\left|p_{n}(y)\right|=\left|q_{n}(x)\right| & \leq\left|T_{n}\left(\frac{b}{2}(x+1)\right)\right|\left\|q_{n}\right\|_{[-1,1]} \\
& =\left|T_{n}\left(\frac{b}{2}(x+1)\right)\right|\left\|p_{n}\right\|_{[0, b]} .
\end{aligned}
$$

Note that

$$
\begin{aligned}
\max _{x \in[b, b+\delta]}\left|T_{n}\left(\frac{b}{2}(x+1)\right)\right| & =\max _{x \in\left[1,1+2 \frac{\delta}{b}\right]}\left|T_{n}(x)\right| \\
& =\left\|T_{n}\right\|_{\left[1,1+2 \frac{\delta}{b}\right]}
\end{aligned}
$$

This clearly implies

$$
\left\|p_{n}\right\|_{[b, b+\delta]} \leq\left\|T_{n}\right\|_{\left[1,1+2 \frac{\delta}{b}\right]}\left\|p_{n}\right\|_{[0, b]} .
$$

Using inequality (41) above, we see that

$$
\left\|T_{n}\right\|_{\left[1,1+\frac{\delta}{b}\right]} \leq\left(1+2 \frac{\delta}{b}\left(1+\sqrt{1+\frac{b}{\delta}}\right)\right)^{n}
$$

The result now follows by letting $k_{b, \delta}=2 \frac{\delta}{b}\left(1+\sqrt{1+\frac{b}{\delta}}\right)>0$ and observing that

$$
\begin{aligned}
\left\|p_{n}\right\|_{[0, b+\delta]} & =\max \left\{\left\|p_{n}\right\|_{[0, b]},\left\|p_{n}\right\|_{[b, b+\delta]}\right\} \\
& \leq \max \left\{\left\|p_{n}\right\|_{[0, b]},\left(1+k_{b, \delta}\right)^{n}\left\|p_{n}\right\|_{[0, b]}\right\} \\
& =\left(1+k_{b, \delta}\right)^{n}\left\|p_{n}\right\|_{[0, b]} .
\end{aligned}
$$


Using this inequality, we also get that, for $b, \delta>0$ fixed,

$$
\left\|p_{n}\right\|_{[0, b]} \geq\left\|p_{n}\right\|_{[0, b-\delta]}\left(\frac{1}{1+k_{b-\delta, \delta}}\right)^{n} .
$$

Note that also $\lim _{\delta \rightarrow 0} k_{b-\delta, \delta}=0$.

We can now use this to prove

Theorem 1. The function $t_{M}(x)$ is continuous on $(0, \infty)$.

Proof. First, note that $t_{M}(x)$ is (non-strictly) increasing in $x$. Let $b \in(0, \infty)$, $\epsilon>0$ and choose $\delta=\min \left\{\delta_{1}, \delta_{2}\right\}$, where $\delta_{1}$ is chosen such that $k_{b, \delta_{1}}<\frac{\epsilon}{t_{M}(b)}$ and $\delta_{2}$ is such that $\frac{k_{b-\delta_{2}, \delta_{2}}}{1+k_{b-\delta_{2}, \delta_{2}}}<\frac{\epsilon}{t_{M}(b)}$.

Let $0<|b-x|<\delta$. The argument splits into two cases:

(1) Suppose that $0<b-x<\delta \leq \delta_{1}$. Since $t_{M}(x)$ is increasing, we have

$$
\begin{aligned}
0 \leq t_{M}(x)-t_{M}(b) & \leq t_{M}\left(b+\delta_{1}\right)-t_{M}(b) \\
& =\lim _{n \rightarrow \infty}\left(\inf _{p_{n} \in \mathcal{M}_{n}[x]}\left\|p_{n}\right\|_{\left[0, b+\delta_{1}\right]}^{1 / n}-\inf _{p_{n} \in \mathcal{M}_{n}[x]}\left\|p_{n}\right\|_{[0, b]}^{1 / n}\right) \\
& \leq \lim _{n \rightarrow \infty}\left(\inf _{p_{n} \in \mathcal{M}_{n}[x]} k_{b, \delta_{1}}\left\|p_{n}\right\|_{[0, b]}^{1 / n}\right) \\
& =t_{M}(b) k_{b, \delta_{1}}<\epsilon
\end{aligned}
$$

(2) Now, assume $0<x-b<\delta \leq \delta_{2}$. Here, we get

$$
\begin{aligned}
0 \leq t_{M}(b)-t_{M}(x) & \leq t_{M}(b)-t_{M}\left(b-\delta_{2}\right) \\
& =\lim _{n \rightarrow \infty}\left(\inf _{p_{n} \in \mathcal{M}_{n}[x]}\left\|p_{n}\right\|_{[0, b]}^{1 / n}-\inf _{p_{n} \in \mathcal{M}_{n}[x]}\left\|p_{n}\right\|_{\left[0, b-\delta_{2}\right]}^{1 / n}\right) \\
& \leq \lim _{n \rightarrow \infty}\left(\inf _{p_{n} \in \mathcal{M}_{n}[x]}\left(\frac{k_{b-\delta_{2}, \delta_{2}}}{1+k_{b-\delta_{2}, \delta_{2}}}\right)\left\|p_{n}\right\|_{[0, b]}^{1 / n}\right) \\
& =t_{M}(b) \frac{k_{b-\delta_{2}, \delta_{2}}}{1+k_{b-\delta_{2}, \delta_{2}}}<\epsilon
\end{aligned}
$$

Thus, for $0<|b-x|<\delta$, we have $\left|t_{M}(b)-t_{M}(x)\right|<\epsilon$ for any $b \in(0, \infty)$, proving continuity for $x>0$.

As mentioned before, Borwein and Erdélyi stated this result for the nonmonic transfinite diameter. In fact, if $\mathcal{A}_{n}[x] \subseteq \mathbb{R}_{n}[x]$ and

$$
t_{\mathcal{A}}(I)=\lim _{n \rightarrow \infty} \inf _{0 \neq p_{n} \in \mathcal{A}_{n}[x]}\left\|p_{n}\right\|_{I}^{\frac{1}{n}},
$$

one can define $t_{\mathcal{A}}(x)$ in the equivalent way and the prove continuity of this function for $x \geq 0$ as in Theorem 1 .

The continuity gives some answers to conjectures made by Hare and Smyth in [4]:

Conjecture 1 (Zero-endpoint interval conjecture). If $I=[0, b]$ with $b \leq 1$, then $t_{M}(I)=\frac{1}{n}$, where $n=\max \left(2,\left\lceil\frac{1}{b}\right\rceil\right)$ is the smallest integer $n \geq 2$ for which $\frac{1}{n} \leq b$. 
Conjecture 2 (Maximal obstruction implies $t_{M}(I)$ conjecture). If an interval $I$ of length less than 4 has a maximal obstruction $m(I)$, then $t_{M}(I)=m(I)$.

Theorem[1 clearly shows Conjecture 1 to be false: As $t_{M}(x)$ is continuous to the left of $\frac{1}{n}, n \in \mathbb{N}$, we cannot have $t_{M}([0, b])=\frac{1}{n+1}$ for all $b<\frac{1}{n}$, as claimed in the conjecture. A further implication is that for these intervals, $t_{M}(I) \neq m(I)$, contrary to Conjecture 2

It turns out that $t_{M}(x)$ is indeed constant on a large interval to the right of $\frac{1}{n+1}, n \in \mathbb{N}$. We define for $n>1 \in \mathbb{N}$,

$$
b_{\max }(n)=\sup _{b>\frac{1}{n}}\left\{b \mid t_{M}(b)=\frac{1}{n}\right\} .
$$

For $n=1$, this quantity is not finite, as $t_{M}(I)=1$ for $|I| \geq 4$ (see $\underline{3}$ for details). For $n=2$, we can use the results in [4] to obtain $1.26 \leq b_{\max }(2)<$ 1.328. For $n>2$, we have the following:

Theorem 2. Let $n>2 \in \mathbb{N}$. Then

$$
\frac{1}{n}+\frac{1}{n^{2}(n-1)} \quad<b_{\max }(n) \leq \frac{4 n}{(2 n-1)^{2}} .
$$

Proof. The first inequality follows from the polynomial

$$
P_{n}(x)=x^{n^{2}-2}\left(x^{2}-n x+1\right) .
$$

This polynomial was first used in [4] and shown to satisfy the following:

1. $P_{n}\left(\frac{1}{n}\right)=\left(\frac{1}{n}\right)^{n^{2}}$

2. $P_{n}^{\prime}\left(\frac{1}{n}\right)=0$ and the polynomial has no other extrema in $\left[0, \frac{1}{n-1}\right]$.

3. $P_{n}(x)$ has a root $\beta_{n}=\frac{2}{n+\sqrt{n^{2}-4}}>\frac{1}{n}$ and $\left|P_{n}(x)\right|$ is strictly increasing in $\left(\beta_{n}, \frac{1}{n-1}\right)$.

The authors used this to show that $\left\|P_{n}\right\|_{\left[0, \frac{1}{n}+\epsilon\right]}=\left(\frac{1}{n}\right)^{n^{2}}$ for some $\epsilon>0$.

Evaluating $P_{n}(x)$ at $x=\frac{1}{n}+\frac{1}{n^{2}(n-1)}$ gives

$$
\left|P_{n}\left(\frac{1}{n}+\frac{1}{n^{2}(n-1)}\right)\right|=\left(\frac{n^{2}-n+1}{n^{2}-n}\right)^{n^{2}} \frac{n^{3}-3 n^{2}+2 n-1}{\left(n^{2}-n+1\right)^{2}}
$$

To show that this is indeed less than $\left(\frac{1}{n}\right)^{n^{2}}$, first note that the sequence

$$
\left\{\left(\frac{n^{2}-n}{n^{2}-n+1}\right)^{n^{2}}\right\}_{n=1}^{\infty}
$$

is increasing and tends to $e^{-1}$. As it is increasing, we clearly have

$$
\left(\frac{n^{2}-n}{n^{2}-n+1}\right)^{n^{2}} \geq\left(\frac{2}{3}\right)^{4} \text { for } n>2
$$


Further, note that for all $n$,

$$
\left(\frac{2}{3}\right)^{4}>\frac{n^{3}-3 n^{2}+2 n-1}{\left(n^{2}-n+1\right)^{2}}
$$

Thus taking (10) and (11) together, we have, for $n>2$,

$$
\left(\frac{n^{2}-n}{n^{2}-n+1}\right)^{n^{2}}>\frac{n^{3}-3 n^{2}+2 n-1}{\left(n^{2}-n+1\right)^{2}} .
$$

Rearranging now gives the desired result.

For the upper bound, one has to look directly at (6). Suppose we have some $p_{d}(x) \in \mathcal{M}_{d}[x]$ such that $\left\|p_{d}\right\|_{I_{\delta_{n}}}^{\frac{1}{d}}=\frac{1}{n}$ on an interval $I_{\delta_{n}}=\left[0, \frac{1}{n-1}-\delta_{n}\right]$. Clearly, $\left\|p_{d}\right\|_{\left[0, \frac{1}{n-1}\right]}^{\frac{1}{d}} \geq \frac{1}{n-1}$ since $\frac{1}{n-1} \leq t_{M}\left(\left[0, \frac{1}{n-1}\right]\right)$. Thus, using ([6]), we get

$$
\frac{1}{n-1} \leq \frac{1}{n}\left(1+k_{\frac{1}{n-1}-\delta_{n}, \delta_{n}}\right)
$$

Using the explicit expression for $k_{\frac{1}{n-1}}-\delta_{n}, \delta_{n}$ obtained in the proof of lemma 3. we see that then $\delta_{n} \geq \delta_{\min }(n)=\frac{1}{4 n^{3}-8 n^{2}+5 n-1}$, thus obtaining

$$
b_{\max }(n) \leq \frac{1}{n-1}-\delta_{\min }(n)=\frac{4 n}{(2 n-1)^{2}}
$$

Using the computational methods outlined in Section 3, we get improved lower bounds for $n=3, \ldots 8$ (see Table 1):

$$
\begin{array}{ll}
0.465 \leq b_{\max }(3), & 0.303 \leq b_{\max }(4) \\
0.230 \leq b_{\max }(5), & 0.184 \leq b_{\max }(6) \\
0.148 \leq b_{\max }(7), & 0.130 \leq b_{\max }(8)
\end{array}
$$

Note that the first two polynomials already appeared in [4. While the second polynomial was taken directly from the article, the first, due to Kevin Hare, is replacing the polynomial quoted in the article, which did not attain the maximal obstruction on the interval. As $n$ gets larger, computations become increasingly difficult, as the difference $\frac{1}{n-1}-\frac{1}{n}$ tends to zero.

Using (6), one can obtain a new lower bound for $t_{M}([0, b]), b<1$ :

Lemma 4. Let $I_{b}=[0, b], b<1$ and let $n=\min \left\{m \in \mathbb{N} \mid \frac{1}{m}>b\right\}$. Then

$$
t_{M}\left(I_{b}\right) \geq \max \left\{\frac{1}{n+1}, \frac{b}{2(1+\sqrt{1-n b})-n b}\right\}
$$

Proof. Let $\delta=\frac{1}{n}-b$. As can easily be seen from (6),

$$
\begin{aligned}
t_{M}\left(\left[0, \frac{1}{n}-\delta\right]\right) & \geq t_{M}\left(\left[0, \frac{1}{n}\right]\right) \frac{1}{1+k_{\frac{1}{n}-\delta, \delta}} \\
& =\frac{1-n \delta}{n(1+n \delta+2 \sqrt{n \delta})} \\
& =\frac{b}{2-n b+2 \sqrt{1-n b}}
\end{aligned}
$$


Seeing that, for $b \leq b_{\max }(n+1), \frac{1}{n+1}$ is a larger lower bound, we get the result.

Another open conjecture, this one taken from [3], is the following:

Conjecture 3 (Farey Interval Conjecture). Let $\frac{p}{q}, \frac{r}{s} \in \mathbb{Q}$ with $q, s>0$ be such that $r q-p s=1$. Then

$$
t_{M}\left(\left[\frac{p}{q}, \frac{r}{s}\right]\right)=\max \left\{\frac{1}{q}, \frac{1}{s}\right\}
$$

Computationally, the authors verified the conjecture for denominators up to 21 and it was proved for an infinite family of such intervals in 4 . It is perhaps worth noting that continuity of $t_{M}(x)$ cannot be used to find a counterexample to this conjecture, as the following argument shows:

Let $n>1 \in \mathbb{N}$. We will show that we cannot find a Farey Interval of the form $\left[\frac{k}{n}, \frac{p}{q}\right]$ with $1 \leq k<n, n=\min \{q, n\}$ and $\frac{p}{q}>b_{\max }^{*}(n)$, where

$$
b_{\max }^{*}(n)=\sup _{\frac{k}{n}<b}\left\{b \mid t_{M}\left(\left[\frac{k}{n}, b\right]\right)=\frac{1}{n}\right\}
$$

As can easily be derived from the proof of Lemma 3

$$
b_{\max }^{*}(n) \leq \frac{k\left(4 n^{2}+1\right)}{n(2 n-1)^{2}} .
$$

If we wanted to use this to derive a counterexample to the Farey Interval Conjecture, we would need $\frac{p}{q}>b_{\max }^{*}(n)$. Using the Farey property

$$
p n-q k=1,
$$

we can write this as

$$
\frac{1+q k}{q k}>\frac{4 n^{2}+1}{(2 n-1)^{2}}
$$

From this it follows that $1+q k<2 n+1$, so that, using (12) again, $1+q k=2 n$. Now, as $q>n$, it is clear that $k=1$ for this to hold.

In the case $k=1$, one can show that the Farey interval is then of the form $\left[\frac{1}{n}, \frac{1+t}{(1+t) n-1}\right], t \in \mathbb{N}$. But no such interval with $\frac{1+t}{(1+t) n-1}>b_{\max }^{*}(n)=\frac{4 n^{2}+1}{(2 n-1)^{2} n}$ exists.

The result for the remaining Farey intervals is obtained by using the transformations $x \rightarrow m \pm x, m \in \mathbb{Z}$.

\section{Computational Methods}

In order to improve the lower bounds for $b_{\max }(n)$ attained in Theorem 2, we need to turn to computational methods to attempt to find a monic polynomial $P(x) \in \mathbb{Z}[x]$ attaining the maximal obstruction. Computational methods are split into two parts:

1. Using a modification of the LLL algorithm to find factors $f_{i}(x)$ of $P(x)$. 
2. Using Linear Programming methods first used in 2 in connection with the integer transfinite diameter with additional equality constraints obtained in 4 to determine the exponents $\alpha_{i}$.

We will briefly discuss the implementations of both parts of the algorithm.

1. LLL is an algorithm that, given a basis $\mathbf{b}$ for a lattice $\Lambda$, produces a "small" basis for $\Lambda$ with respect to a given inner product $\langle\cdot, \cdot\rangle$. In their modification of the LLL algorithm for monic polynomials introduced in [3, the authors used the Lattice $\mathbb{Z}_{n}[x]$ with the basis $\mathbf{b}=\left(1, x, x^{2}, \cdots x^{n}\right)$ and the inner product

$$
\left\langle p_{n}, q_{n}\right\rangle=\int_{a}^{b} p_{n}(x) q_{n}(x) d x+a_{n} b_{n}
$$

for $p_{n}(x)=a_{n} x^{n}+\cdots+a_{0}, q_{n}(x)=b_{n} x^{n}+\cdots+b_{0} \in \mathbb{Z}_{n}[x]$. The additional factor $a_{n} b_{n}$ is used to discourage non-monic factors from appearing, and the algorithm usually produces only one monic basis element of degree $n$. In practice, we used the following recursive algorithm to identify factors $f_{i}(x)$ of $P(x)$ for an interval $I=[a, b]$ where the maximal obstruction polynomial $q(x)=a_{d} x^{d}+\cdots+a_{0}$ is known:

(a) Start with $\mathbf{b}=\left(1, x, x^{2}, \cdots, x^{k}\right)$ for $k=20$ (in some cases, a larger basis was required initially).

(b) Run LLL, generating a list of factors $l=\left\{f_{i}(x)\right\}$.

(c) Sieve the list by using the condition that if $f_{i}(x) \mid P(x)$, then the resultant has to satisfy $\left|\operatorname{Res}\left(f_{i}, q\right)\right|=1$ (see [4]).

(d) For every $f_{i}$ still in $l$, define $\widehat{\mathbf{b}_{i}}=\left(1, f_{i}(x), f_{i}(x) x, f_{i}(x) x^{2}, \cdots f_{i}(x) x^{k}\right)$ and re-run the LLL-Algorithm with this basis, adding new factors to $l$.

(e) Repeat steps (a)-(d) until no more new factors are found, at which point we return $l$.

2. To determine the exponents $\alpha_{i}$ of $f_{i}(x), 1 \leq i \leq N$, we use a technique first used by Borwein and Erdélyi in [2]. Given a list of factors $l=\left\{f_{i}(x)\right\}$, one attempts to minimise $m$ subject to

$$
\begin{cases}\sum_{i=1}^{N} \frac{\alpha_{i}}{\operatorname{deg} f_{i}} \log \left|f_{i}(x)\right| \leq m-g(x) & x \in X \\ \sum_{i=1}^{N} \alpha_{i}=1 & \\ \sum_{i=1}^{N} \frac{\alpha_{i}}{\operatorname{deg} f_{i}} \frac{f^{\prime}\left(\beta_{s}\right)}{f\left(\beta_{s}\right)}=0 & q\left(\beta_{s}\right)=0,1 \leq s \leq \operatorname{deg} q \\ \alpha_{i} \geq 0 & 1 \leq i \leq N\end{cases}
$$

over a finite set $X \subset I$. Here, $g(x)$ is a function such that

$$
g(x)= \begin{cases}0 & q(x)=0 \\ \epsilon(x)>0 & q(x) \neq 0 .\end{cases}
$$

The use of this function is theoretically not necessary, but is useful when doing computations, as avoids having to deal with exact values at points where the polynomial does not need to attain the maximal obstruction. 
The first two constraints in (13) are taken from 2 with a slight modification to the first, while the third is unique to the monic case and taken from 4. This is also where we get the final set of constraints:

Let $\beta_{s}$ be a root of $q(x)$ and define $\hat{f}_{i}^{(s)}=\frac{1}{\operatorname{deg} f_{i}} \log \left|f_{i}\left(\beta_{s}\right)\right|$. If $b_{1}=$ $-\frac{1}{d} \log \left|a_{d}\right|, b_{2}, \cdots b_{l}$ is an independent generating set for the $\mathbb{Z}$-lattice generated by $-\frac{1}{d} \log \left|a_{d}\right|$ and the $\hat{f}_{i}^{(s)}$, let $c_{j, i}^{(s)}$ be such that

$$
\sum_{j=1}^{l} c_{j, i}^{(s)} b_{j}=\hat{f}_{i}^{(s)}
$$

Then we get the additional conditions, derived in [4]:

$$
\sum_{i=1}^{N} c_{j, i}^{(s)} \alpha_{i}=\left\{\begin{array}{rl}
-\frac{1}{\operatorname{deg} q} & j=1 \\
0 & j>1
\end{array}, 1 \leq s \leq \operatorname{deg} q\right.
$$

Again, we use a recursive algorithm for determining the exponents. Given a set of points $X_{k}$, we use (13) and (14) to determine the optimal exponents $\left\{\alpha_{1}^{(k)}, \alpha_{2}^{(k)}, \ldots, \alpha_{N}^{(k)}\right\}$ attaining the minimum value $m_{k}$. Then, we construct the normalised "polynomial"

$$
P^{(k)}(x)=\prod_{i=1}^{N} f_{i}(x)^{\frac{\alpha_{i}^{(k)}}{\operatorname{deg} f_{i}}}
$$

and add its extrema to $X_{k}$ to obtain $X_{k+1}$. Starting with a small set of values $X_{1} \subset I$, we repeat this procedure until we get $K \in \mathbb{N}$ such that $\left|m_{K}-m_{K-1}\right|<\epsilon$ for required precision $\epsilon>0$.

Finally, we compute the supremum norm of $P_{K}(x) \approx e^{m_{K}}$ on the interval and verify that $\left\|P_{K}\right\|_{I}=\left|a_{d}\right|^{-\frac{1}{d}}$. One can attempt to find rational approximations of smaller denominator to the exponents, always checking that the obstruction is still attained. The attaining polynomial $P(x)$ is then found by clearing denominators in the exponents of $P_{K}(x)$.

The author would like to thank Kevin Hare for useful insights and Chris Smyth for guidance and relentless proofreading. Further, I would like to thank the referee for carefully reading the manuscript and catching some mistakes as well as providing useful comments. 
Table 1: Polynomials used for lower bounds on $b_{\max }(n)$

\begin{tabular}{|c|c|}
\hline$n$ & $P_{n}(x)$ \\
\hline 3 & $\begin{array}{l}x^{45944640}\left(x^{14}-11406261 x^{13}+47054086 x^{12}-88456310 x^{11}\right. \\
+100247244 x^{10}-76341256 x^{9}+41208853 x^{8}-16202606 x^{7}+4692047 x^{6}-999261 x^{5} \\
\left.+154318 x^{4}-16766 x^{3}+1211 x^{2}-52 x+1\right)^{2450525} \\
\left(x^{8}+14184 x^{7}-34944 x^{6}+36442 x^{5}-20832 x^{4}+7041 x^{3}-1405 x^{2}+153 x-7\right)^{877415} \\
\left(x^{8}+4842 x^{7}-10935 x^{6}+10355 x^{5}-5317 x^{4}+1594 x^{3}-278 x^{2}+26 x-1\right)^{2571030} \\
\left(x^{8}+7812 x^{7}-18072 x^{6}+17561 x^{5}-9271 x^{4}+2864 x^{3}-516 x^{2}+50 x-2\right)^{595980} \\
\left(x^{7}-1233 x^{6}+2406 x^{5}-1913 x^{4}+791 x^{3}-179 x^{2}+21 x-1\right)^{1210840} \\
\left(x^{5}-3 x^{4}+7 x^{3}-11 x^{2}+6 x-1\right)^{1052898}\end{array}$ \\
\hline 4 & $\begin{array}{l}x^{640}\left(x^{5}+432 x^{4}-456 x^{3}+179 x^{2}-31 x+2\right)^{47} \\
\left(x^{7}+8760 x^{6}-13342 x^{5}+8488 x^{4}-2784 x^{3}+514 x^{2}-50 x+2\right)^{35}\end{array}$ \\
\hline 5 & $\begin{array}{l}x^{1050990}\left(x^{10}+5544095 x^{9}-9115714 x^{8}+6623719 x^{7}-2790988 x^{6}\right. \\
\left.+751349 x^{5}-133974 x^{4}+15818 x^{3}-1192 x^{2}+52 x-1\right)^{78796} \\
\left(x^{6}+4950 x^{5}-4605 x^{4}+1698 x^{3}-310 x^{2}+28 x-1\right)^{21825}\end{array}$ \\
\hline 6 & $\begin{array}{l}x^{5232473}\left(x^{5}+1260 x^{4}-852 x^{3}+215 x^{2}-24 x+1\right)^{118824} \\
\left(x^{7}-140190 x^{6}+132517 x^{5}-51966 x^{4}+10819 x^{3}-1261 x^{2}+78 x-2\right)^{200917}\end{array}$ \\
\hline 7 & $x^{44}\left(x^{5}+3472 x^{4}-1826 x^{3}+358 x^{2}-31 x+1\right)$ \\
\hline 8 & $x^{12288}\left(x^{2}-8 x+1\right)^{246}\left(x^{4}-576 x^{3}+208 x^{2}-25 x+1\right)^{741}$ \\
\hline
\end{tabular}

\section{References}

[1] Borwein, P., And Erdélyi, T. Polynomials and Polynomial Inequalities. Springer, New York, NY, 1995.

[2] Borwein, P., And Erdélyi, T. The integer Chebyshev problem. Math. Comp. 65, 214 (1996), 661-681.

[3] Borwein, P. B., Pinner, C. G., And Pritsker, I. E. Monic integer Chebyshev problem. Math. Comp. 72, 244 (2003), 1901-1916.

[4] Hare, K., And Smyth, C. The monic integer transfinite diameter. Math. Comp. 75 (2006), 1997-2019. 